\title{
The Gravitational Waves Speed Is Much More Than the Speed of Light
}

\author{
Hadi Ensan \\ Technical College, Technical and Vocational University, Neyshaboor, Iran
}

Email address:

h_ensan1@yahoo.ca

\section{To cite this article:}

Hadi Ensan. The Gravitational Waves Speed Is Much More Than the Speed of Light. American Journal of Astronomy and Astrophysics. Vol. 4, No. 5, 2016, pp. 54-59. doi: 10.11648/j.ajaa.20160405.11

Received: May 24, 2016; Accepted: August 10, 2016; Published: August 25, 2016

\begin{abstract}
This paper is the theory for the speed of gravitational waves and comparison of it with the speed of light. The GW (Gravitational Waves) have been observed [1] and the role of dark energy and cold dark matter are being studied [2,3], but the key role of gravitational waves speed $\left(c_{G W}\right)$ in the stability of cosmic formations has not been studied much. LIGO discovered the gravitational waves (2015). General relativity predicts for a strong gravitational lens that light and GW travel at the same speed. Blas et al. (2016) showed that the ratio of the speeds of gravitational waves and of light is $c_{G W} / c_{\gamma}<1.7$. Moore and Nelson (2001) showed that: $1-{ }^{c_{G W}} / c_{\gamma}<10^{-15}$, etc. [2]. As is discussed here gravitational waves speed should be much more than the speed of light. Nowadays, almost all of the instruments that are used for studying in Astronomy and Astrophysics are some how related to the applications of radio frequency rays, infra red, visible light, ultra violet rays, X-rays and Gama rays that in general name are called electromagnetic waves. The light speed in vacuum; $c_{\gamma} \cong 3 \times 10^{8} \mathrm{~m} / \mathrm{s}$ is the highest speed that so far has been known in Physics [4]. Comparing the speed of light to the cosmic distances and using it as a communication tool, it looks like the speed of snail on the ground. Gravity is one of important forces responsible for relative stability of galaxies, solar systems, planetary systems, etc. By discovering, producing, transmitting and receiving the gravitation waves like the knowledge that exists about electromagnetic waves now, it could be a great help for very fast communication tool and also new big and clear window, etc. to the Universe. But before that, at first it should be studied whether the speed of gravity field propagation is much more that the speed of light or not.
\end{abstract}

Keywords: Gravitational Waves Speed, Comparison of Light with Gravity

\section{Introduction}

The distances in the most cosmic ranges are so huge that the scientists introduced light-year unit and also the cosmic distance scale, $1 A U \approx 1.5 \times 10^{11} \mathrm{~m}$ as the astronomical unit [5]. Newton (1642-1727) discovered the law of gravity [6] that attracts the masses to each other. Watching the images of stars and the other space formations like cluster of galaxies about 3 billion light-year away [7] brings us the doubt that: If very huge masses and very far distances from each other are controlled by gravity forces and the signal speed of gravity is as the same speed of light, The very low speed of light compared to the sizes of cosmic distances is not enough for maintaining such formations not to collapse. As it is shown in this paper, the speed of light is indirectly compared with the speed of gravitational field. Then the role of gravitational waves speed for relative stability of the galaxies mentioned. By reviewing this paper, we will consider that $c_{G w} \gg c_{\gamma}$.

\section{Procedure}

For discussion of the subject, it is mentioned three reasons. In the first reason the Doppler Effect $[8,9]$ is reviewed, and then based on that effect the speed of light is compared to the speed of gravitational waves. The conclusion is that, the $c_{G W}$ should be more that the speed of light. In the Reason two, by mentioning the Black Holes [10] that they do not let the light rays to be emitted and comparing again the two speed of light and gravitational waves based on the Doppler Effect it is concluded that $c_{G W}$ should be more that the speed of light. In the reason three, the relative stability of a galaxy less, more similar to our galaxy is discussed and the exchange number of gravitons per unit of masses per unit of time $\mathrm{N}$, is 
introduced for the first time:

$$
N=\frac{n_{g} v_{g}}{m_{1} m_{2} r}
$$

Where $n_{g}$, is the total number of gravitons exchange between the two masses at the time of $\mathrm{t}$ and $v_{g}$ is $c_{G W}$. As it is considered here if $c_{G W} \leq c_{\gamma}$, then they galaxies could collapse so faster compared to their life span and there should not be conservation of galaxy system any more. Every place that the word of speed used, it means the abstract velocity relative to the mentioned point.

\subsection{Reason One}

Comparing the speed of gravitational waves to the speed of light using the Doppler Effect, as we already know,

According to the Doppler Effect:

The observed frequency of a sound is raised if the separation distance, that is, the distance between an observer and the source of the sound, is shortening, and is lowered if the separation distance is increasing [8].

A different situation exists if the source is stationary and the listener moving. Suppose that the listener is moving towards the source. Because of the motion, the listener will run into some of the crests which would not otherwise have yet reached the listener's ears. As a result, a listener approaches the source will hear a frequency larger than $f$. Or, if the listener is running away from the source, a frequency less than $f$ will hear will be heard. Indeed, if the listener is moving away with the speed of sound, only the accompanying crest will influence the listener's ear. In effect, the frequency heard is reduced to zero.

Let us examine this phenomenon from a quantitative point of view. We define the following:

$v_{w}=$ velocity of wave

$v_{L}=$ velocity of listener

$v_{S}=$ velocity of source

$f^{\prime}=$ Frequency heard when listener is moving respect with to the source

$f=$ Frequency heard when listener is still respect with to the source

$$
\begin{gathered}
\frac{f^{\prime}}{f}=\frac{v_{w}-v_{L}}{v_{w}-v_{S}}=\frac{1-\left(v_{L} / v_{w}\right)}{1-\left(v_{S} / v_{w}\right)} \\
\frac{f^{\prime}}{f}=\frac{\text { speed of wave relative to listener }}{\text { speed of wave relative to source }}
\end{gathered}
$$

This form shows clearly that $f^{\prime}$ is increased if the listener is moving against the waves and is decreased if the source is moving in the direction of the waves. This general effect is called the Doppler Effect after Christian Johann Doppler, who showed in 1842 that this effect should be observed for sound and light waves [8]. However, that (2) is not correct for light waves when the source or observer is moving with speeds close to the speed of light. The difficulty arises because of the relativity principle, which states that the speed of light in vacuum is independent of the motion of the observer or the light source.) For light the result of calculation from [9] is:

$$
f^{\prime}=f \frac{\sqrt{1-\frac{v^{2}}{c^{2}}}}{1-\frac{v}{C}}
$$

The conclusion is that if the relative velocity of observer be more that the speed of the sound wave, the source of the sound has no transferring mechanical energy or force to the observer. In the simple words, if the relative speed of observer and the sound source $\left|v_{L}-v_{S}\right|$ be more than the velocity of wave $v_{w}$, the observer will be free from any effect of upcoming waves. One of the results is: If there exists a sound source with the conditions of:

1) The power of the sound is enough to reach to the stationary observer with the distance of $\mathrm{x}$.

2) There is not any obstacle like sound absorber between the sound source and observer.

3) The observer has instruments to receive the sound energy from the source at distance of $\mathrm{x}$. But, there is no sound reception.

We can conclude that the relative speed of observer and the sound source $\left|v_{L}-v_{w}\right|$ is more than the velocity of waves $v_{s}$.

Now we examine that conclusion, for comparing the speed of light $v_{l}$ waves and the speed of gravitational waves $c_{G W}$.

We can arrange (1) to the new form of:

$$
\frac{f^{\prime}}{f}=\frac{v_{\text {gravity }}-v_{\text {listener }}}{v_{\text {gravity }}-v_{\text {source }}}=\frac{1-\left(v_{\text {listener }} / v_{\text {gravity }}\right)}{1-\left(v_{\text {source }} / v_{\text {gravity }}\right)}
$$

Defining the following:

$$
v_{\text {gravity }}=C_{G W}
$$

$v_{\text {listener }}=$ velocity of object or listener travelling with speed of light

$v_{\text {source }}=$ velocity the source or mass that emits gravity waves

$\frac{f^{\prime}}{f}=\frac{\text { speed of gravitational waves relative to the speed of light }}{\text { speed of gravitational waves relative to the object }}$

Imagine an object or an observer travels with the speed of light relative to another object (It is a science fiction case now). According to the above conclusion, if its speed (observer speed) that is the speed of light be more than $c_{G W}$ emitting from another object as a source, then there could not be any effect of interaction between the object (gravitational source) and observer (light).

In reality there exists effect or interaction between light and gravitation force.

The deflection of a ray of straight by the sun's gravitational field happens during a total solar eclipse, when the sun's glare does not blind the observers. The star can be viewed and displaced from its true position. The deviation is attributed to the bending of the light rays by sun [11]. When the hunter chases the hunt, he can catch it, only if his speed is more than it has. In another words the speed of light that is influenced by gravity, is less than the speed of gravitational waves. 


\subsection{Reason Two}

Black Holes: The correct criterion for a star to be a black hole turns out to be $G M / c^{2} R \geq \frac{1}{2}$. The Schwarzschild radius $R_{S}$ of a body of mass $\mathrm{M}$ is defined as: $R_{S}=\frac{2 G M}{c^{2}}$ [12].

They do not let the light rays to be emitted and the conclusion could be that $c_{G W}>c_{\gamma}$, because:

The follower never reaches to the escaper if the follower speed is less than the latter. In the sound example, a listener that travels with the speed more than the speed of sound, from the sound source could never hear the sound signals emitting from the sound source.

Suppose the light is escaper and the gravitation force be the follower. If the speed of gravitational waves be less than or equal to the speed of light, then if a mass moves with $c_{\gamma}$, it would not be affected by field of gravity (Although by Physics laws it is impossible that particles with non zero masses at rest could move with the speed of light). Light quantum mass at rest is zero. Even if their stationary quantum masses were not zero, they could not be affected by field of gravitational force.

If the propagation speed of gravity waves was less than or equal to the speed of light, then according to the Doppler Effect the Black Hole gravity field could not have influence to the emitting of light. Result:

The speed of gravitational waves is more than the speed of light.

\subsection{Reason Three: Conservation and Relative Stability of Galaxies System}

In the universe for large system of objects and materials like galaxies, Newton Gravity Law $\overrightarrow{\boldsymbol{F}}=-G \frac{m_{1} m_{2}}{r^{3}} \overrightarrow{\boldsymbol{r}}$ is not enough to maintain the relative stability of system, but also speed of communications has a big role. Let us consider an example for sound speed and relation between the source and the receiver.

\subsubsection{The Role of Communication Speed}

Referring to the figure 1, suppose we have a sound source of A that emits towards to receiver of B with enough distance that could receive mechanical energy from the source. The receiver of $\mathrm{B}$ could reflect a part of energy as the form of:

$$
\Delta P_{B, \text { reflection }}=\frac{P_{\text {source }}}{4 \pi R^{2}} a_{B} \cdot k_{r}
$$

It is defined the following:

$\Delta P_{B, \text { reflection }}=$ Some part of mechanical sound

power that reflects by object of $B$

$P_{\text {source }}=$ The sound power of source A

$R=$ Distance of $\mathrm{B}$ from $\mathrm{A}$

$a_{B}=$ Cross-sectional area of $\mathrm{B}$ perpendicular to $\mathrm{R}$

$k_{r}=$ Sound reflection coefficient by B between 0 to 1

Supposing the reflection and the absorption coefficient be the same. Some part of this energy that will be received again by the source of A could be as the form of:

$$
\Delta P_{B \rightarrow A}=\Delta P_{B, \text { reflection }} \cdot \frac{a_{A}}{4 \pi R^{2}}=P_{\text {source }} \cdot \frac{a_{A} \cdot a_{B} \cdot K_{r}}{\left(4 \pi R^{2}\right)^{2}}
$$

Where:

$a_{A}=$ Cross-sectional area of body of A perpendicular to R

If the source of A could recognize this amount of energy then it could "feel" or "hear" the object of B. The equation shows that $\Delta P_{B \rightarrow A}$ is reverse proportional to the quadratic $\left(1 / R^{4}\right.$ of $\mathrm{R}$, the distance between two bodies. In the above example with doubling the distance of two bodies the reflecting power decreases to the $1 / 16$ of power source of $A$ body. If body of A is on Moon and body of B is on Earth, for very low speed of sound with compare to such distance, the messages are almost dead. (In reality, first, there is not such powerful loudspeaker and second, the sound for propagation needs media that does not exist between Earth and Moon, etc.). For maintaining the communication between two bodies not only all mentioned parameters have roles, but also:

The speed of communication has a big role for maintaining the mass formations especially when the distances among them increase.

H. Yukawa believes attractive and repulsive forces can both arise from particle exchange [12]. Graviton has been named as particle exchange for gravity force.

\subsubsection{The Relation Between the Acting Force of Gravity and the Speed of Gravitational Field}

The formula is given here for the first time is defined as below:

$$
n_{g}=N M m t
$$

In SI System, where:

$n_{g}=$ Total exchange number of gravitons for the period time of $t$ is needed to maintain the body of $m$ at a certain orbit with radius of $r$.

$\mathrm{N}=$ The exchange number of gravitons per unit of masses per unit of time that is needed to maintain the body of $m$ at a certain orbit with radius of $r$.

$M$, the bigger mass, $m$, smaller mass and $\mathrm{t}$ is the time.

Equation (7) may be rewritten to the form of:

$$
N=\frac{n_{g}}{M m t}
$$

Assuming gravitational waves has constant speed, $v_{g}$ in vacuum, since $r$ is given by:

$$
r=v_{g} t
$$

Substituting t from (9) into (8) yields:

$$
N=\frac{n_{g}}{M m \frac{r}{v_{g}}}
$$

or:

$$
N=\frac{n_{g} v_{g}}{M m r}
$$

$v_{g}=$ The gravitational waves speed in vacuum

Equation (11) shows relation between gravitons exchange 
and the speed of gravitational waves.

When the orbit of the body of $m$ changes from the radius of $r_{1}$ to the orbit with the radius of $r_{2}$, assuming that the number of gravitons to be exchanged is proportional to the reverse of $r$. If the number of gravitons to be exchanged would be proportional of $r$ or higher power of $r$, then the radius of solar systems or galaxies would be infinitive that in reality they are not. Therefore:

$$
\frac{n_{1}}{n_{2}}=\frac{r_{2}}{r_{1}}
$$

Therefore, we have:

$$
\begin{gathered}
n_{1}=N_{1} M m \frac{r_{1}}{v_{g}} \\
n_{2}=N_{2} M m \frac{r_{2}}{v_{g}}
\end{gathered}
$$

Dividing the sides of (13) to the sides of (14) yields:

$$
\frac{n_{1}}{n_{2}}=\frac{N_{1}}{N_{2}} \frac{r_{1}}{r_{2}}
$$

Now, substituting from (12) into (15)

We have:

$$
\frac{N_{1}}{N_{2}}=\frac{r_{2}^{2}}{r_{1}^{2}}
$$

From (11) for the radiuses of $r_{1}$ and $r_{2}$ we can have:

$$
\begin{gathered}
N_{1}=\frac{n_{1 g} v_{g}}{M m r_{1}} \\
N_{2}=\frac{n_{2 g} v_{g}}{M m r_{2}}
\end{gathered}
$$

Right side of equation of (16) is familiar for us, because it is the same as the ratio of two gravity forces on two different radiuses:

$$
\begin{aligned}
& F_{1}=G \frac{M m}{r_{1}{ }^{2}} \\
& F_{2}=G \frac{M m}{r_{2}{ }^{2}}
\end{aligned}
$$

Dividing the sides of (19) to the sides of (20) yields us:

$$
\frac{F_{1}}{F_{2}}=\frac{r_{2}^{2}}{r_{1}^{2}}
$$

Comparing (21) and (16) gives that:

$$
\frac{F_{1}}{F_{2}}=\frac{N_{1}}{N_{2}}
$$

This shows that assumption of (7) gives familiar gravity formula form and the conclusions could be that:

The gravity force has relation with the speed of gravitational waves, because exchange number of gravitons has relation with the speed of gravitational waves (22), (17) and (18).

The farer masses receive lesser number of gravitons per unit of masses per unit of time (17) and (18).

The gravity force exerting between two masses is proportional to the numbers of gravitons exchange (22).

\subsubsection{One of the Results of Communication Low Speed is the Longer Time for the Signal Receipt}

For the forces like gravity it is needed the bodies continuously evaluate each other. In the sound example, if the sound was communication tool between Earth and Moon, for sending and receiving any message between them, we should wait about 36 days. This time is more than the time is needed for full revolution of Moon (about 29 days) around Earth.

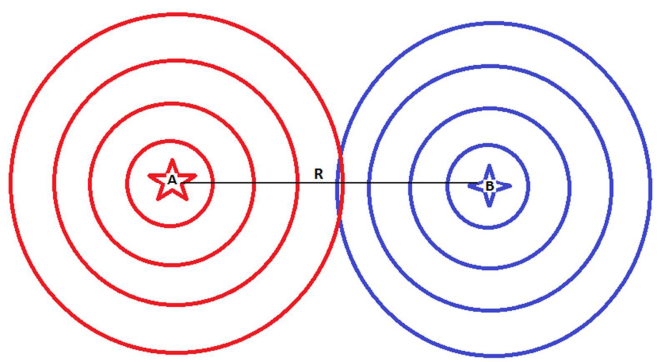

Figure 1. Body of A sends an acoustic signal to the surrounding area (crests are shown by the red circles). It spreads to the spherical surface of $4 \pi R^{2}$ to reach $B$. Energy received by $B$ is small fraction of sound energy emitted by $A$. The reflection of sound by body of $B$ (crests are shown by the blue circles) comes back to $A$, spreads again to the spherical surface of $4 \pi R^{2}$. From (6), the energy received by $A$ from the sound reflection of $B$ is very small fraction of primary energy of signal $A$ and proportional to $1 / R^{4}$.

\subsubsection{Gravitation Field Speed and Conservation of the Galaxies}

Based on consideration of this part, it is concluded again that the gravity force responsible for maintaining the galaxies not to collapse like Milky Way galaxy [13] with about 50,000 light-years radius should have the speed much more than the speed of light.

In galaxies, based on our existing knowledge, the gravitation force of black hole in the center is responsible for maintaining the galaxy not to collapse. Assuming the radius of our galaxy be about 50,000 light-years. This is the distance of the most far stars or masses from black hole.

At first, assuming that the speed of the gravity force waves be (less or) equal to speed of light in the vacuum. Considering a star at the edge of the galaxy (Figure 2) and we have advance technology to trace the beam of the gravitational wave. For the forces like gravity it is needed the bodies continuously evaluate each other. Supposing the precise clock has been adjusted to zero when the black hole send a signal of gravitation to evaluate (The amount of force should exert to hold that star on its orbit.) and then sending enough gravitons ( particles responsible for gravitation field) to that star on the circle or ellipsoid orbit of the edge of the galaxy. When signal reaches to star, the clock shows the passage time of 50,000 years. The star also sends a signal that returned back to black hole after another 50,000 years. Since exchanging of each message in this example takes about 100,000 years, it is expected that at least two processes will happen at this long period of time:

(1) The masses especially, the mass of black hole and star will be decreased because of changing the matter based on Conservation of Mass and Energy Law for any closed system 
to different kinds of energy of (radiation, electromagnetic, thermal, mechanical, gravity, etc.)

(2) The positions of masses specially, the stars at the edge of galaxy change respect with black hole. They continue to move in straight lines based on Newton's Fist Law.

Let us $r_{1}$ be the first distance at the time of reaching the first signal, then the next signal should reaches to the distant of $r_{2}$ (figure 3 ) that the latter is bigger that the first distant:

$$
r_{2}=\sqrt{r_{1}^{2}+\mathrm{d}_{1}^{2}}
$$

Where:

$r_{2}=$ The second distant of star to the center of the galaxy after period time of 100,000 years

$d_{1}=$ The distant that star travels at the straight line free of gravity force perpendicular to $r_{1}$ within the period time of 100,000 years

The third distance $r_{3}$ will be bigger than $r_{2}$ :

$$
r_{3}=\sqrt{r_{2}^{2}+d_{2}^{2}}
$$

Where:

$r_{3}=$ The third distant of star to the center of the galaxy after another period time of 100,000 years

$d_{2}=$ The distant that star travels at the straight line free of gravity force perpendicular to $r_{2}$ within the period time of 100,000 years

Considering the Figure 2, it is concluded that:

$$
r_{n}>r_{n-1}>\cdots>r_{3}>r_{2}>r_{1}
$$

The radius of the galaxy rapidly becomes more longer than it normally happens for the life span of the galaxy. Its shape instead of the approximate circle will be like a cyclone.

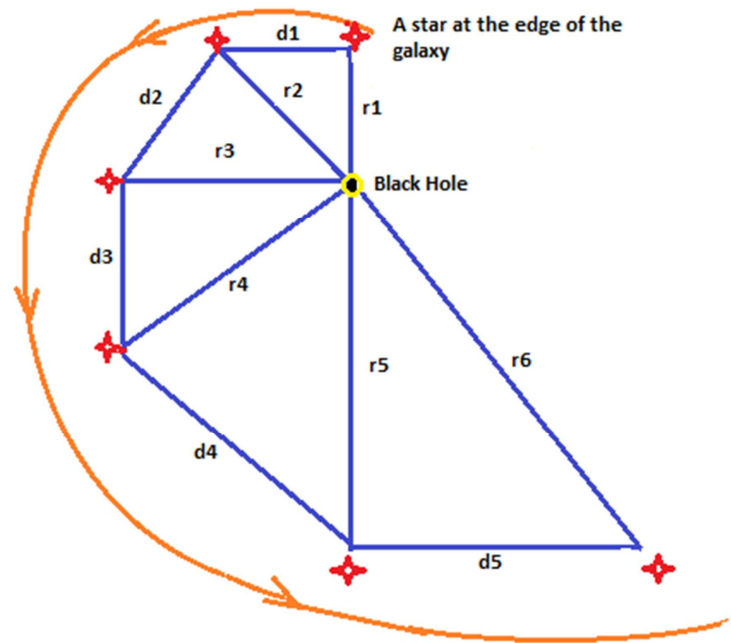

Figure 2. A star at the edge of the galaxy needs maximum time to reach it the gravitational waves from black hole at the center. Drawing shows the result of low speed of gravitational waves speed (the speed of light or less than of it). The radius of galaxy rapidly becomes more longer than it normally happens for the life span of the galaxy. Its shape instead of the circle will be like the cyclone.

The result of such low speed of gravitational waves (speed of light or less than it) will be the fast expanding and collapsing the galaxies.

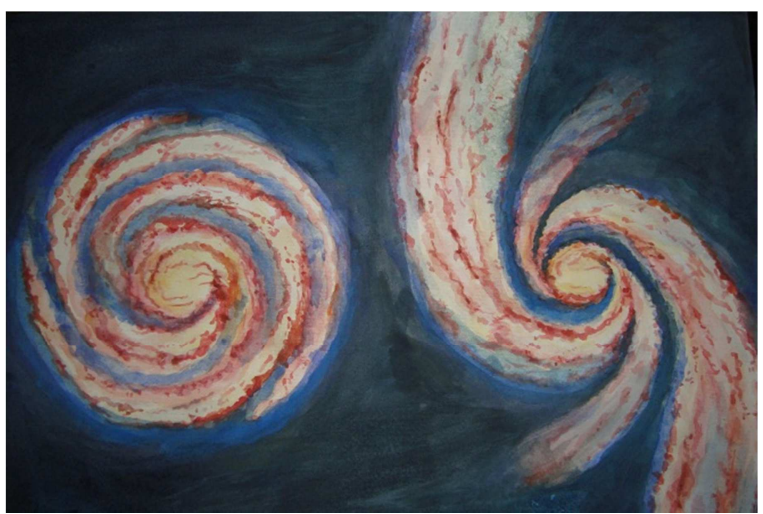

Figure 3. Painted by author Ensan, Comparison for the speed of light and gravitational waves speed. The left figure is the approximate shape of an existing elliptical galaxy; right is the imaginary shape of the same galaxy, if the gravitational propagation speed be low like the speed of light or less. In that case, gravity force can not properly hold the galaxy masses from preventing to expand fast, and then the destruction of galaxy will occur.

If that condition would exist, the space images of the galaxies should show almost homogeny of mix stars at the remaining of old galaxies. The observation of super cluster galaxies at 3 billions light-year away does not show such destructions or collapses (Figure 4).

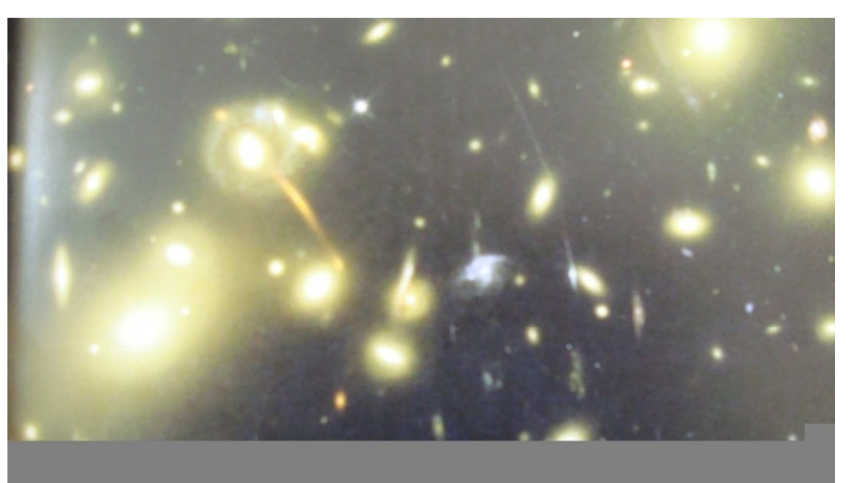

Figure 4. Abell 2218 is a cluster of galaxies some 3 billion light-years away. It is so massive that the gravity of its galaxies is bending the light of galaxies behind it into a series of arcs - an effect known as gravitational lensing [7].

Therefore, if the stars moving around the outer orbits that remain almost the circle shape, then based on the above example, it yields that the speed of gravity force field with the dimensions of galaxies should be so higher than the speed of light to maintain such stability.

\section{Summary and Conclusion}

In this paper by mentioning 3 reasons is shown that: The speed of gravitational propagation speed should be much more than the speed of light. In the reason one; $c_{G W}$ has been compared with $c_{\gamma}$ by using Doppler Effect and the bending effect of light beam near the massive objects like our sun. In the reason two, by mentioning Black Holes that they do not let the light beam emit from conservation of galaxies 
mentioned and explained that one of the reasons for preventing the light not to escape from them is the higher speed of gravitational waves respect with to light speed. In reason three, it is shown that for maintaining the communication between two bodies not only all mentioned parameters have roles, but also the speed of communication has a key role, especially when the distance between them increases. A new formula is introduced for indirect relation between gravity force and its speed: $\mathrm{N}=\frac{n_{g} v_{g}}{M m r}$. Finally the relative stability of galaxies is reviewed and it is resulted the much higher speed of gravitational waves $\left({ }^{c_{G W}} / c_{\gamma} \gg 1\right)$ compared to the speed of light.

\section{References}

[1] B. P. Abbott et al. Observation of Gravitational Waves from a Binary Black Hole Merger, (LIGO Scientific Collaboration and Virgo Collaborations, PHYSICAL REVIEW LETTERS, 2016) https://physics.aps.org/featured-articlepdf/10.1103/PhysRevLett.116.061102

[2] T. E. Collet et al., Testing the Speed of Gravitational Waves over Cosmological Distances with Strong Gravitational Lensing, (University of Portsmouth, UK, 2016) https://arxiv.org/pdf/1602.05882.pdf

[3] M. S. El Naschie, On Quantum Gravity Fractal Space time Equation: $\mathrm{QRG} \cong \mathrm{HD}+\mathrm{FG}$ and its Applications To Dark
Energy, University of Alexandria, Egypt, 2016, http://file.scirp.org/pdf/JMP_2016042816054450.pdf

[4] P. A. Tipler, PHYSICS FOR SCIENTISTS AND ENGINEERS, Forth Edition (W. H. Freeman and Company, New York, USA, 1999) p1036-1039

[5] M. Harwit, Astrophysical Concepts, Third Edition, (SpringerVerlag, USA, 2000) p49

[6] F. J. Bueche, Principles of Physics, (McGraw-Hill International Editions, Singapore, 1988) p70

[7] M. A. Garlick, ASTRONOMY, A visual guide, Firefly Books Ltd. China, 2004), p183

[8] Frederick J. Bueche, Introduction to Physics for Scientists and Engineers, (McGraw-Hill, Singapore, 1986) p585-590

[9] E. Jones, R. Childers, PHYSICS, Contemporary College, (McGraw Hill, USA, 2001) p807-808

[10] D. A. Ostlie, B. W. Carrol, An Introduction to: Modern Stellar Astrophysics, Weber State University, (Addison-Wesley Publishing Company, USA, 1996) p661-663

[11] J. I. Pfeffer, S. Nir, Modern Physics, An Introductory Test, (Imperial College Press, Singapore, 2000) p130

[12] A. Beiser, CONCEPTS OF MODERN PHYSICS, Fourth Edition, (McGraw-Hill International Editions) p434, p83

[13] T. Ferris, GALAXIES, A Sierra Club Book, (Workman Publishing Company, Japan, 1982) p61 